hep-ph/9902404

\title{
Unitarity bound for the single-helicity-flip amplitude in elastic $p p$-scattering.
}

\author{
S. M. Troshin and N. E. Tyurin \\ Institute for High Energy Physics, \\ Protvino, Moscow Region, 142284 Russia
}

\begin{abstract}
On the basis of the $U$-matrix method of the $s$-channel unitarization, we obtain a new unitarity bound for the single-helicity-flip amplitude $F_{5}$ of elastic pp-scattering at small values of $t$.
\end{abstract}


The size and energy dependence of the hadron helicity-flip amplitude is an interesting problem in the study of the asymptotic properties and the role of spindependent interaction and it is also an important issue in the polarimetry studies based on the Coulomb-Nuclear Interference (CNI) [1]. If the hadronic part of the proton-proton interaction is helicity conserving the CNI analysing power would be then due to the interference between a real electromagnetic helicity-flip amplitude and an imaginary hadronic helicity-nonflip amplitude.

However, the hadronic interaction may not conserve helicity in small angle scattering. Helicity conservation does not follow from QCD in a region where chiral symmetry is spontaneously broken. In Regge theory the Pomeron is usually assumed to be helicity conserving. However this is merely an assumption which seems to be wrong; recent experimental results on the central production of mesons and observation of nontrivial azimuthal dependence demonstrates the nonzero helicity tranfer by the effective Pomeron [2]. Earlier it was shown that the unitarity generates different phases in the helicity-flip and nonflip Pomeron contributions [3] and leads to nonzero analysing power.

The CNI analysing power would also certainly change if there were any nonzero hadronic single-helicity-flip amplitude.

The above issues are considered in the recent survey [1]. Among the new results, the most interesting one is the unexpected bound for the single-helicityflip amplitude $F_{5}$ of elastic $p p$-scattering, i.e. the function

$$
\left.\hat{F}_{5}(s, 0) \equiv \frac{m F_{5}(s, t)}{\sqrt{-t}}\right|_{t=0}
$$

cannot increase at $s \rightarrow 0$ faster than

$$
c s \ln ^{3} s,
$$

while for the helicity nonflip amplitudes there is the Froissart-Martin bound $c s \ln ^{2} s$.

In this note we show that, in fact, a stronger bound for the function $\hat{F}_{5}(s, 0)$ can be obtained if one takes into account the unitarity in the explicit way, e.g. uses an unitarization method based on the $U$-matrix approach [4].

This method is based on the unitary representation for helicity amplitudes of elastic $p p$-scattering:

$$
F_{\lambda_{1}, \lambda_{2}, \lambda_{3}, \lambda_{4}}(s, b)=U_{\lambda_{1}, \lambda_{2}, \lambda_{3}, \lambda_{4}}(s, b)+i \rho(s) \sum_{\lambda^{\prime}, \lambda^{\prime \prime}} U_{\lambda_{1}, \lambda_{2}, \lambda^{\prime}, \lambda^{\prime \prime}}(s, b) F_{\lambda^{\prime}, \lambda^{\prime \prime}, \lambda_{3}, \lambda_{4}}(s, b),
$$


where $\lambda^{\prime} s$ are the intial and final proton's helicities. $F_{i}$ are the helicity amplitudes in the standard notations, i.e.

$$
F_{1} \equiv F_{1 / 2,1 / 2,1 / 2,1 / 2}, F_{2} \equiv F_{1 / 2,1 / 2,-1 / 2,-1 / 2}, F_{3} \equiv F_{1 / 2,-1 / 2,1 / 2,-1 / 2}
$$

and

$$
F_{4} \equiv F_{1 / 2,-1 / 2,-1 / 2,1 / 2}, F_{5} \equiv F_{1 / 2,1 / 2,1 / 2,-1 / 2} .
$$

The kinematical function $\rho(s) \simeq 1$ at $s \gg 4 m^{2}$ and will be neglected in the following.

The functions $U_{i}(s, b)$ could be treated similar to the eikonal, i. e. they can be considered as input amplitudes.

Explicit solution of Eqs. (1) has the following form:

$$
\begin{aligned}
& F_{1}(s, b)=\frac{\tilde{U}_{1}(s, b)\left[1-i U_{1}(s, b)\right]-i \tilde{U}_{2}(s, b) U_{2}(s, b)}{\left[1-i U_{1}(s, b)\right]^{2}-\left[U_{2}(s, b)\right]^{2}} \\
& F_{3}(s, b)=\frac{\tilde{U}_{3}(s, b)\left[1-i U_{3}(s, b)\right]-i \tilde{U}_{4}(s, b) U_{3}(s, b)}{\left[1-i U_{3}(s, b)\right]^{2}-\left[U_{4}(s, b)\right]^{2}}
\end{aligned}
$$

where

$$
\tilde{U}_{i}(s, b)=U_{i}(s, b)+2 U_{5}(s, b) F_{5}(s, b)
$$

and

$$
F_{5}(s, b)=\frac{U_{5}(s, b)}{\left[1-i U_{1}(s, b)-i U_{2}(s, b)\right]\left[1-i U_{3}(s, b)-i U_{4}(s, b)\right]-4 U_{5}^{2}(s, b)} .
$$

We consider the two cases. First, we suppose that the helicity nonflip functions $U_{1}(s, b)$ and $U_{3}(s, b)$ are the dominant ones.

In this case one can get [3]

$$
F_{5}(s, t)=\frac{s}{\pi^{2}} \int_{0}^{\infty} b d b \frac{U_{5}(s, b)}{\left[1-i U_{1}(s, b)\right]\left[1-i U_{3}(s, b)\right]} J_{1}(b \sqrt{-t}) .
$$

Unitarity requires that $\operatorname{Im} U_{1,3}(s, b) \geq 0$. The functions $U_{1,3}(s, b)$ could be different. For our purposes, however, it is safe to assume that they are the same $U_{1}(s, b)=U_{3}(s, b)=U(s, b)$. For the function $U(s, b)$ we use a simple form

$$
U(s, b)=g s^{\Delta} e^{-\mu b} .
$$


This is a rather general parameterization for $U(s, b)$ which provides correct analytical properties in the complex $t$-plane, i.e. it is consistent with the spectral representation for the function $U(s, b)$ [5]:

$$
U(s, b)=\frac{\pi^{2}}{s} \int_{t_{0}}^{\infty} \rho(s, t) K_{0}(b \sqrt{t}) d t .
$$

We do not use here model features and do not consider detailed structure of the helicity functions $U_{i}$ but appeal to reasonable arguments of the general nature. To maximize the function $U_{5}(s, b)$ we take it in the form $U_{5}(s, b)=a U(s, b)$ where $|a|<1$. Then from Eq. (2) it follows that at $s \rightarrow \infty$ :

$$
\left|\hat{F}_{5}(s, 0)\right| \leq c s \ln ^{2} s .
$$

This means that the magnitude of the ratio

$$
r_{5}(s, 0) \equiv 2 \hat{F}_{5}(s, 0) /\left[F_{1}(s, 0)+F_{3}(s, 0)\right]
$$

cannot increase with energy and will not exceed constant at $s \rightarrow \infty$.

This result has a general meaning and retains in the opposite case, i.e. in the case when the function $U_{5}(s, b)$ is a dominant one. We have for the amplitude $F_{5}(s, t)$ the following representation

$$
F_{5}(s, t)=\frac{s}{\pi^{2}} \int_{0}^{\infty} b d b \frac{U_{5}(s, b)}{1-4 U_{5}^{2}(s, b)} J_{1}(b \sqrt{-t}) .
$$

Using for $U_{5}(s, b)$ the functional dependence in the form of Eq. (3) it can be easily shown that the same bound Eq. (5) does take place for the single-helicityflip amplitude $\hat{F}_{5}$.

Thus we can state that general principles do not allow rising behavior of $\left|r_{5}(s, 0)\right|$. The experimental data as well as the most of the model predictions are consistent with this bound [6, 7]. This result allow us to hope that the contribution of the single-helicity-flip amplitude could be controllable and the effective use of the CNI polarimeter would be possible.

Above results were obtained in impact parameter representation for simplicity. They can easily be reproduced using the partial wave expansion.

It is accurate account of the unitarity for the helicity amplitudes leads to Eq. (5), i.e. due to unitarity the amplitude $F_{5}(s, b)$ has a peripheral dependence on the variable $b$ at high energy and

$$
\left|F_{5}(s, b=0)\right| \rightarrow 0
$$


at $s \rightarrow \infty$. This is a consequence of the explicit unitarity representation for the helicity amplitudes and it means that the assumption on $F_{5}(s, b)=$ constant at $b<R(s)$ [1] appears to be inadequte (however, it remains to be good for the helicity-nonflip amplitudes).

Thus, as it was shown, we have an asymptotic bound

$$
\left|r_{5}(s, 0)\right| \leq \text { constant }
$$

at $s \rightarrow \infty$.

To conclude, it is worth to note that only the model-dependent estimations exist for the magnitude of the ratio $\left|r_{5}(s, 0)\right|$, but the rise of the function $\left|r_{5}(s, 0)\right|$ at $s \rightarrow \infty$ can be excluded on the unitarity ground.

\section{References}

[1] N.H. Buttimore et al., BNL-HET 98/46, CPT-98/P.3693, hep-ph/9901339 and references therein.

[2] F. E. Close and G. A. Schuler, CERN-TH/99-28; hep-ph/9902243 and references therein.

[3] S. M. Troshin and N. E. Tyurin, Spin Phenomena in Particle Interactions, World Scientific, Singapore, 1994.

[4] A. A. Logunov, V. I. Savrin, N. E. Tyurin, O. A. Khrustalev, Teor. Math. Phys. 6 (1971) 157.

[5] S. M. Troshin and N. E. Tyurin, Theor. Math. Phys. 50 (1982) 150.

[6] T. L. Trueman, CNI Polarimetry and the hadronic spin dependence of $p$ p scattering, Proceedings of the 12th International Symposium on High Energy Spin Physics, Amsterdam, Sept. 1996, SPIN96, Eds. C.W. de Jager, et al., World Scientific, Singapore, 1997, p. 833.

[7] A. D. Krisch and S. M. Troshin, Estimate of elastic proton-proton polarization at small $P_{T}^{2}$ near $1 \mathrm{TeV}$, Proceedings of SPIN96, p.830. 